\title{
Avaliação da prática de vigilância epidemiológica nos serviços públicos de saúde no Brasil*
}

\author{
Assessment of epidemiological surveillance practice in the public health \\ services of Brazil
}

\author{
Marilia Sá Carvalho**, Keyla B. F. Marzocchi***
}

\begin{abstract}
CARVALHO, M.S. \& MARZOCCHI, K.B.F. Avaliação da prática de vigilância epidemiológica nos serviços públicos de saúde no Brasil. Rev. Saúde públ., S. Paulo, 26: 66-74, 1992. São apresentados os resultados da avaliação de processo das atividades de vigilância epidemiológica, realizada em 1985, em 948 unidades de saúde situadas em 98 dos mais populosos municípios de cada Estado brasileiro. Foram analisados os seguintes aspectos: fluxo de informações, análise de dados e realização de investigação epidemiológica. Foram considerados potencialmente determinantes do desempenho: inserção institucional, atividades de vacinação, aspectos gerenciais e capacitação em serviço. A análise estatística bascou-sc na análise de correspondência múltipla e na classificação hierárquica ascendente, disponíveis no programa "Systeme Portable Pur L' Analise De Données SPAD". As unidades avaliadas não apresentaram padrão uniforme de desempenho, sendo classificadas em seis grupos segundo a atuação na vigilância epidemiológica. Em $53,7 \%$ das unidades foi observado desrespeito às normas mais elementares das atividades de vigilância epidemiológica. A presença de alividades de vacinação nas unidades estava relacionada com um melhor desempenho em vigilância epidemiológica. Foi apontada a necessidade de rever o modelo de vigilância epidemiológica ainda em uso no país, pois não é mais concebível a redução da epidemiologia dos serviços de saúde às doenças transmissíveis ou o gerenciamento dos serviços e programas sem a informação epidemiológica.
\end{abstract}

Descritores: Vigilância epidemiológica. Avaliação de programas. Imunização.

\section{Introdução}

Relatos de problemas para o bom descmpenho da vigilância epidemiológica vêm sc repetindo ao longo de décadas. A subnotificação, a falta de recursos humanos capacitados e as dificuldades na integração entre as diversas atividades do controle de doenças são constataçōes presentes em variados documentos oficiais ${ }^{6,7,10}$.

* Trabalho realizado com financiamento da Secretaria Nacional de Af̧ões Básicas em Saúde do Ministério de Saúde. Resumo da dissertação de mestrado, subordinada ao mesmo título, apresentada por Marília Sá Carvalho à Escola Nacional de Saúde Pública, em 1990. Apresentado no $1^{2}$ Congresso Brasileiro de Epidemiologia, Campinas, 1990.

* Departamento de Epidemiologia e Métodos Quantitativos da Escola Nacional de Saúde Pública da Fundação Oswaldo Cruz - Rio de Janeiro, RJ - Brasil.

*** Departamento de Ciĉncias Biológicas da Escola Nacional de Saúde Pública/FIOCRUZ - Rio de Janciro, RJ - Brasil.

Separatas/Reprints: M.S. Carvalho - Rua Leopoldo Bulhões, 1480/8 andar - Manguinhos - 21041 - Rio de Janciro, RJ - Brasil.
A partir da década de 80 , com o objetivo de avançar na superação dos entraves verificados, foram implementadas, entre outras atividades, elaboração e/ou revisão de normas técnicas, treinamentos c supervisões 8 . 9 . Em 1983, com recursos oriundos do FINSOCIAL, foi estruturado ambicioso programa de treinamento em vigilância epidemiológica, com base em materiais instrucionais desenvolvidos pela Secretaria de Recursos Humanos do Minislério da Saúde - o "Curso de Introdução à Vigilância Epidemiológica" (CIVE) - e pela Escola Nacional de Saúde Pública (ENSP) - o "Curso Básico de Vigilância Epidemiológica" (CBVE). Dois anos após, haviam sido treinados aproximadamente três mil profissionais de nível superior com o CBVE e outros tantos com o CIVE, conforme o cadastro de egressos organizado pela Secretaria Nacional de Açõcs Básicas de Saúde (SNABS) do Ministćrio da Saúdc. Foi então proposto ao Grupo de Apoio ao Programa Ampliado de Imunizaçōes (PAI), na ENSP, que avaliasse junto aos Estados os rcsultados de referido processo de capacitação de recursos humanos $\mathrm{em}$ vigilância epidemiológica.

No presente artigo foi analisada a prática em vigilância cpidemiológica das unidades da rede 
pública de assistência à saúde do país, buscando uma melhor compreensão dos desempenhos observados. Outras atividades avaliadas, como o programa de imunizações, somente serão apresentadas quando diretamente relacionadas à vigilância epidemiológica*.

Os desdobramentos que se esperava de tão ampla avaliação, infelizmente, não ocorreram. $O$ relatório ${ }^{3}$ encaminhado aos Estados não teve retorno. O próprio Ministério, com as transferências de técnicos e mudanças de chefias aparentemente se desinteressou. Espera-se que a divulgação dos presentes achados venha a contribuir para a melhoria do sistema de vigilância epidemiológica implantado no país.

\section{Metodologia}

São avaliadas a estrutura e o processo de ações normatizadas da vigilância epidemiológica, que são executadas, ao menos sob forma simplificada, em todo o território nacional. Foram visitados 98 municípios, sendo incluído sempre as capitais e, em seguida, os de maior população em cada Estado ${ }^{4}$.

Em cada município foram avaliadas as unidades de atendimento ambulatorial que contavam com médico no seu quadro de pessoal e pertencentes a alguma das seguintes instituiçőes: Sccretaria Estadual de Saúde (SES), Secretaria Municipal de Saúde (SMS) ou Instituto Nacional de Assistência Médica e Previdência Social (INAMPS). Acrescentou-se a essas unidades, os hospitais com maior número de leitos de pediatria em cada município selecionado, independente da instituição mantenedora. Assim sendo, deve-se observar que não é possível a generalização estatística das conclusões obtidas para os municípios não-visitados, pois a seleção destes não foi aleatória. Entrclanto, a equipe que trabalhou no projeto e que discutiu os resultados descritivos apresentados no relatório da pesquisa ${ }^{3}$ não encontrou razão para acreditar que os demais municípios do mesmo porte tivessem, $\mathrm{em}$ geral, práticas muito diferentes em relação à vigilância epidemiológica.

As variáveis utilizadas para analisar o desempenho das unidades em vigilância epidemiológica foram selecionadas com base em experiências anteriores $1,12,13$. As variáveis "determinantes" foram selecionadas entre os fatores geralmente accitos como tendo influência sobre o processo, alguns ligados estritamente ao setor, outros relacionados ao funcionamento global dos serviços.

* Informações adicionais poderão ser obtidas junto às autoras.
Foram as seguintes as variáveis selecionadas, em cada aspecto da prática de vigilância epidemiológica:

Fluxo de informações - fontes de informação da unidade para casos de doenças transmissíveis; envio e periodicidade de notificações e envio de noficação negativa.

Registro e Análise da Informaçāo - existência de registro do número de casos de difteria, meningite, sarampo e febre tifóide ocorridos no período de janeiro a outubro de 1985; análise da distribuição dos casos por área geográfica, faixa etária e tempo.

Investigação Epidemiológica - disponibilidade de fichas de investigação epidemiológica de casos de poliomielite, difteria, sarampo e meningite; informação sobre surtos ocorridos.

Em relação às condições potencialmente determinantes do desempenho da unidade em vigilância epidemiológica, considerou-se:

Determinantes Específicos - presença de profissional treinado pelo CBVE; supervisões recebidas em vigilância epidemiológica; disponibilidade de normas escritas de vigilância epidemiológica das principais doenças objeto de controle; existência de boletins epidemiológicos na unidade.

Funcionamento Geral das Unidades - Instituição mantenedora; relação com a comunidade, por meio de conselhos, trabalho extra-muros e/ou órgãos de comunicação de massas; disponibilidade de dados da população e da área de abrangência da unidade; aplicação das vacinas do Programa Nacional de Imunização (PNI); existência de unidade subordinada.

Os dados foram colhidos diretamente nas unidades de saúde, com um questionário do tipo fechado. Cada tipo de instituição incluída no universo foi tratada como um extrato independente. $O$ número de unidades locais amostradas foi definido em função do total de unidades de cada instituição, em cada município. Foram visitadas 948 unidades, sendo 328 das Secretarias Estaduais de Saúde, 300 das Secretarias Municipais de Saúde, 162 do INAMPS e 158 hospitais.

Os dados obtidos foram submetidos à Análise de Correspondência Múltipla e em seguida a uma técnica de classificação automática das unidades 5 . $A$ análise foi feito com o auxílio do "SPAD - Systeme Portable pour l' Analyse des Données"2, conjunto arliculado de programas desenvolvidos especialmente para análise estalística descritiva de grandes tabclas. Foi utilizada a versão de 1985 , cedida pclo Instituto de Matemática Pura e Aplicada - IMPA, para utilização no computador de grande porte do Laboratório Nacional de Computação Científica. Os simbolos de identificação de cada categoria de cada variável no gráfico da análise de correspondência estão apresentados nas Tabelas 1 e 2, e no Anexo. 
A coleta de dados realizou-se entre 11 de novembro e 13 de dezembro de 1985 , em todos as unidades federadas, com exceção do Rio de Janeiro e do Distrito Federal, realizada aproximadamente um mês antes. Os entrevistadores foram recrutados entre os funcionários das próprias Secretarias Estaduais de Saúde, em geral de nível central ou regional, supervisionados diretamente por técnico especialmente designado para tal, dos quadros do Ministério ou da ENSP.

\section{Resultados e Discussão}

As Tabelas 1 e 2 resumem a distribuição das variáveis entre as 948 unidades estudadas.

A análise de correspondência apontou, em linhas gerais, uma relativa coerência no descmpenho das unidades nas atividades avaliadas, conforme pode ser obscrvado na Figura, ou seja, de maneira geral se a unidade notifica corretamente, também nas demais atividades o desempenho estará de acordo com o normatizado.

A variável "Informante" indica as fontes de informação sobre casos de doenças transmissíveis utilizadas na unidade. As quatro calegorias dessa variável, distribuídas ao longo do eixo um (horizontal), desenham uma curva de desempenho que vai da

Tabela 1. Distribuição das unidades segundo variáveis de desempenho da vigilância epidemiológica - Brasil, 1985

\begin{tabular}{|c|c|c|c|c|}
\hline \multirow[t]{2}{*}{ Variável } & \multirow[t]{2}{*}{ Categorias } & \multirow[t]{2}{*}{ Simbolo" } & \multicolumn{2}{|c|}{ Unidades } \\
\hline & & & $N^{2}$ & $\%$ \\
\hline $\begin{array}{l}\text { Informantes } \\
\text { de Doenças } \\
\text { Transmissiveis }\end{array}$ & $\begin{array}{l}\text { Ninguém } \\
\text { Só unidade } \\
\text { Comunidade } \\
\text { Outras unid/ } \\
\text { Ignorado }\end{array}$ & $\begin{array}{l}\text { INFN } \\
\text { INFM } \\
\text { INFG } \\
\text { INFS } \\
\text { INFI }\end{array}$ & $\begin{array}{r}197 \\
420 \\
132 \\
186 \\
13\end{array}$ & $\begin{array}{r}20,8 \\
44,3 \\
13,9 \\
19,6 \\
1,4\end{array}$ \\
\hline $\begin{array}{l}\text { Envio de } \\
\text { Notificação }\end{array}$ & $\begin{array}{l}\text { Correto } \\
\text { Irregular } \\
\text { Não envia } \\
\text { Ignorado } \\
\text { Presente }\end{array}$ & $\begin{array}{l}\text { NOTS } \\
\text { NOTM } \\
\text { NOTN } \\
\text { NOTI } \\
\text { SANA }\end{array}$ & $\begin{array}{r}509 \\
303 \\
124 \\
12 \\
60\end{array}$ & $\begin{array}{r}53,7 \\
32,0 \\
13,1 \\
1,3 \\
6,3\end{array}$ \\
\hline $\begin{array}{l}\text { Análise } \\
\text { de Dados }\end{array}$ & $\begin{array}{l}\text { Ausente } \\
\text { S/registro } \\
\text { Sem casos } \\
\text { Ignorado }\end{array}$ & $\begin{array}{l}\text { NANA } \\
\text { RANA } \\
\text { FANA } \\
\text { IANA }\end{array}$ & $\begin{array}{r}227 \\
602 \\
63 \\
6\end{array}$ & $\begin{array}{r}23,9 \\
63,5 \\
6,6 \\
0,6\end{array}$ \\
\hline $\begin{array}{l}\text { Ficha de } \\
\text { Investigaçāo } \\
\text { Epidemiológica }\end{array}$ & $\begin{array}{l}\text { Presente } \\
\text { Ausente } \\
\text { Sem casos } \\
\text { lgnorado }\end{array}$ & $\begin{array}{l}\text { SFIC } \\
\text { NFIC } \\
\text { FFIC } \\
I F I C\end{array}$ & $\begin{array}{r}93 \\
573 \\
258 \\
24\end{array}$ & $\begin{array}{r}9,8 \\
60,4 \\
27,2 \\
2,5\end{array}$ \\
\hline Total de Unidad & & & 948 & 100,0 \\
\hline
\end{tabular}

* O significado dos símbolos encontra-se no Anexo. pior situação, à direita no gráfico (Figura), até a meIhor. Estas categorias são: nenhuma fonte de informação (INFN); apenas funcionários (médicos e enfermeiros) da própria unidade (INFM); instituições não vinculadas diretamente ao setor saúde, como escolas, creches, associaçōes de moradores, excluindo as que recebem também de outras unidades (INFG); outras unidades de saúde (INFS).

Também a variável "Notificação" apresenta padrão semelhante, formando um gradiente entre ausência de notificação (NOTN), notificação irregular, impontual ou sem notificação negativa (NOTM) e notificação correta (NOTS).

Entretanto, o gradiente observado entre desempenho correto e precário não é acompanhado pelas variáveis "Análise dos Dados" e "Investigação Epidemiológica", ou seja, as atividades que exigcm um grau maior de elaboração, com competência técnica mais especializada, não acompanham o gradiente de desempenho do conjunto de uni-

Tabela 2. Distribuição dos dados segundo determinantes da vigilância epidemiológica

\begin{tabular}{|c|c|c|c|c|}
\hline Variável & Categorias & Símbolo* & Ne & $\%$ \\
\hline Instituição & $\begin{array}{l}\text { Estado } \\
\text { Municipio } \\
\text { INAMPS } \\
\text { Hospital }\end{array}$ & $\begin{array}{l}\text { SES } \\
\text { SMS } \\
\text { INPS } \\
\text { HOSP }\end{array}$ & $\begin{array}{l}328 \\
300 \\
162 \\
158\end{array}$ & $\begin{array}{l}34,6 \\
31,6 \\
17,1 \\
16,7\end{array}$ \\
\hline $\begin{array}{l}\text { Dados } \\
\text { Demográficos }\end{array}$ & $\begin{array}{l}\text { Completos } \\
\text { Incompletos } \\
\text { Nenhum } \\
\text { Ignorado }\end{array}$ & $\begin{array}{l}\text { DEMO } \\
\text { DEMM } \\
\text { DEMN } \\
\text { DEMI }\end{array}$ & $\begin{array}{r}148 \\
310 \\
489 \\
1\end{array}$ & $\begin{array}{r}15,6 \\
32,7 \\
51,6 \\
0,1\end{array}$ \\
\hline $\begin{array}{l}\text { Relação } \\
\text { Comunitária }\end{array}$ & $\begin{array}{l}\text { Variada } \\
\text { Alguma } \\
\text { Nenhuma } \\
\text { lgnorado }\end{array}$ & $\begin{array}{l}\text { COMO } \\
\text { COMM } \\
\text { COMN } \\
\text { COMI }\end{array}$ & $\begin{array}{r}105 \\
359 \\
482 \\
2\end{array}$ & $\begin{array}{r}11,1 \\
37,9 \\
50,8 \\
0,2\end{array}$ \\
\hline PNI & $\begin{array}{l}\text { Presente } \\
\text { Ausente }\end{array}$ & $\begin{array}{l}\text { SPNI } \\
\text { NPNI }\end{array}$ & $\begin{array}{l}660 \\
288\end{array}$ & $\begin{array}{l}69,6 \\
30,4\end{array}$ \\
\hline $\begin{array}{l}\text { Egresso } \\
\text { do CBVE }\end{array}$ & $\begin{array}{l}\text { Presente } \\
\text { Ausente } \\
\text { Ignorado }\end{array}$ & $\begin{array}{l}\text { SCBV } \\
\text { NCBV } \\
\text { ICBV }\end{array}$ & $\begin{array}{l}228 \\
291 \\
429\end{array}$ & $\begin{array}{l}24,1 \\
30,7 \\
45,3\end{array}$ \\
\hline $\begin{array}{l}\text { Supervisāo } \\
\text { em Vigilância } \\
\text { Epidemiológica }\end{array}$ & $\begin{array}{l}\text { Presente } \\
\text { Ausente } \\
\text { Ignorado }\end{array}$ & $\begin{array}{l}\text { SSVE } \\
\text { NSVE } \\
\text { ISVE }\end{array}$ & $\begin{array}{r}163 \\
757 \\
28\end{array}$ & $\begin{array}{r}17,2 \\
79,9 \\
3,0\end{array}$ \\
\hline $\begin{array}{l}\text { Normas } \\
\text { de Vigilância }\end{array}$ & $\begin{array}{l}\text { Presente } \\
\text { Ausente } \\
\text { Ignorado }\end{array}$ & $\begin{array}{l}\text { SNOR } \\
\text { NNOR } \\
\text { INOR }\end{array}$ & $\begin{array}{r}194 \\
732 \\
22\end{array}$ & $\begin{array}{r}20,5 \\
77,2 \\
2,3\end{array}$ \\
\hline $\begin{array}{l}\text { Boletim } \\
\text { Epidemiológico }\end{array}$ & $\begin{array}{l}\text { Presente } \\
\text { Ausente } \\
\text { Ignorado }\end{array}$ & $\begin{array}{l}\text { SBOL } \\
\text { NBOL } \\
\text { IBOL }\end{array}$ & $\begin{array}{r}253 \\
686 \\
9\end{array}$ & $\begin{array}{r}26,7 \\
72,4 \\
0,9\end{array}$ \\
\hline Total de Unidade & & & 948 & 100,0 \\
\hline
\end{tabular}

- O significado dos símbolos encontra-se no Anexo. 
dades. Em relação à análise dos dados, a ausência de registro (RANA) está relacionada ao desempenho precário. As demais categorias, entretanto, não apresentam relações, claramente estabclccidas, com as outras variáveis.

Entre os fatores determinantes, o mais importante é, sem dúvida, a definição de atribuições da unidade, condicionada essencialmente por sua instituição mantenedora.

Na Figura, a localização da categoria "Secretarias Estaduais de Saúde", relativamente mais próximo das categorias indicativas de desempenho adequado, confirma o tradicional papel das unidades da rede estadual nas atividades habituais de saúde pública. A categoria "Secretarias Municipais de Saúde" apresenta-se, em geral, em situação intermediária nos gradientes de desempenho.

O INAMPS e os Hospitais atuam na vigilância epidemiológica e imunizações de forma precária.

Entre as demais variáveis relacionadas ao funcionamento global da unidade, a que apresenta relação mais estreita com o desempenho nas atividades de vigilância epidemiológica é a existência do programa de vacinação. Aparentemente, esta atividade tem efeito positivo sobre as atividades de vigilância epidemiológica, mesmo quando não desempenhada a contento.

O conjunto das atividades consideradas como determinantes específicos é, em gcral, da responsabilidade do nível central das Secretarias Estaduais de Saúde e algumas vezes das Secretarias Municipais de Saúde; é exceção o programa de capacitação apoiado pclo Ministério da Saúde e efetuado sob coordenação do nível central de cada estado, que recebcu verbas e apoio de outras instituições ${ }^{7,10}$.

Em relação à capacitação de pessoal, do conjunto de questões constantes do questionário que tinham como objetivo avaliar precisamente o papel desempenhado pelos cursos de vigilância epidemiológica promovidos pela SNABS, pouco se pode extrair. Em pouquíssimas unidades se obteve informaçōes referentes a cursos freqüentados pelos funcionários. Em relação ao CIVE, onde a inscrição do profissional cra dcfinida pelas chefias e não apenas por interesse particular do funcionário, a desinformação foi também enorme, impossibilitando utilizar cstas variávcis na análise. Em

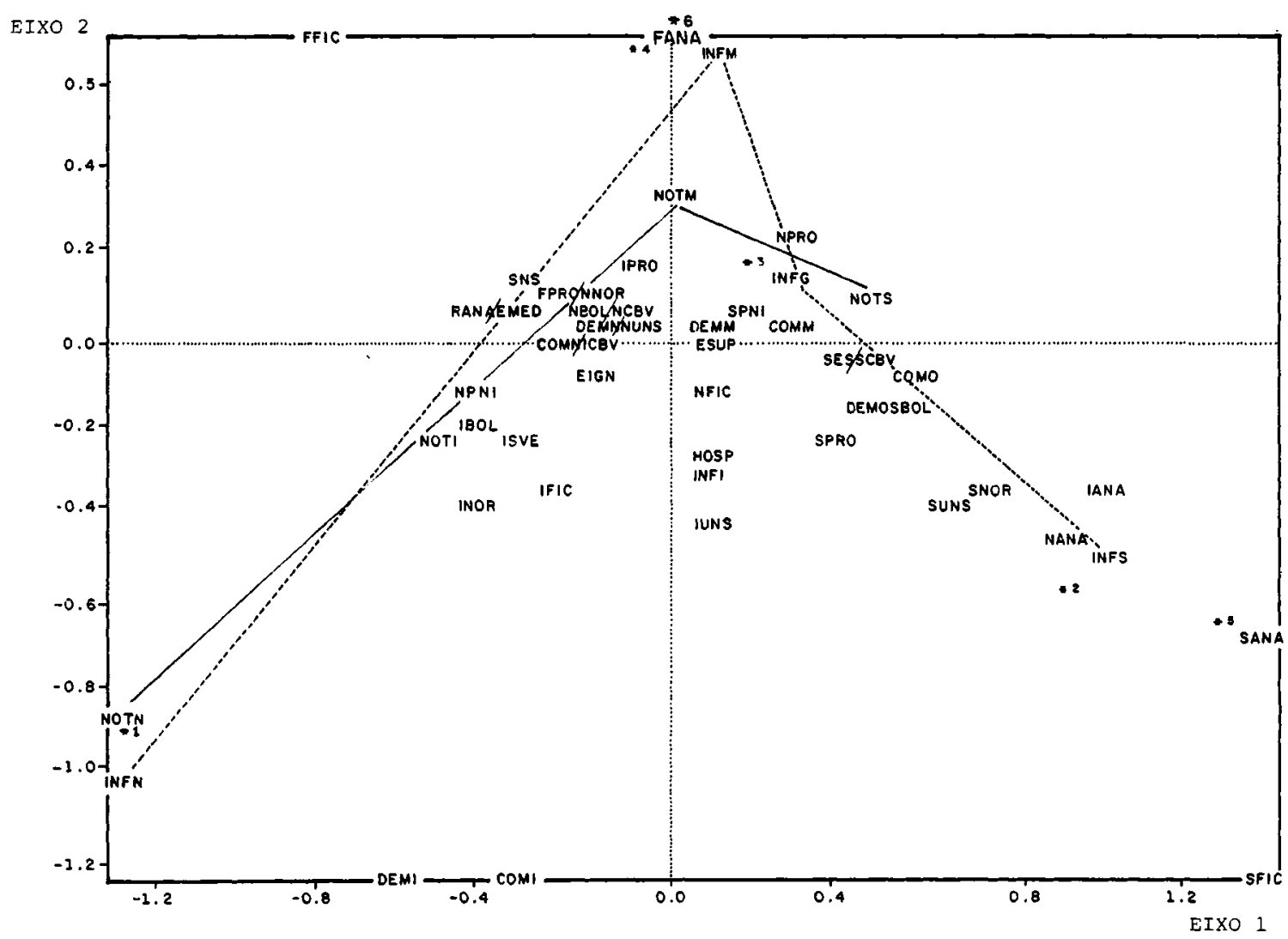

Figura. Análise de correspondência: distribuição gráfica das categorias das variáveis da avaliação. (Listagem emitida pelo "SPAD" - O significado dos símbolos encontra-se no Anexo). 
Tabela 3. Percentual de unidades na classe ${ }^{n *} 1 "$, categorias que as caracterizam e percentual destas categorias no universo total na classe, Brasil, 1985. Classe ${ }^{*}$ - 188 unidades $-19,8 \%$ do total

\begin{tabular}{llrr}
\hline Variável & $\begin{array}{c}\text { Categorias } \\
\text { Caracteristicas }\end{array}$ & $\begin{array}{c}\% \text { no } \\
\text { Universo }\end{array}$ & $\begin{array}{c}\% \text { na } \\
\text { Classe }\end{array}$ \\
\hline Informante & $\begin{array}{l}\text { Nenhum informante } \\
\text { na unidade }\end{array}$ & 20,8 & 90,4 \\
Notificação & $\begin{array}{l}\text { Ausente } \\
\text { Análise de Dados }\end{array}$ & 13,1 & 62,8 \\
PNI & Ausen registro de casos & 63,5 & 96,8 \\
Ativ. Comunitária & Nenhuma & 30,4 & 53,7 \\
Boletim Epid. & Ausente & 50,8 & 73,4 \\
Normas & Ausente & 72,4 & 89,4 \\
Investigação Epid. Nega existência de & 77,2 & 88,3 \\
& casos & 27,2 & 39,3 \\
Dados & Ausentes & 51,6 & 64,4 \\
Demográficos & & & \\
Instituição & Municipio & 31,6 & 42,0 \\
CBVE & Não dispõe de & & \\
& informação & 45,3 & 55,3 \\
Instituição & INAMPS & 17,1 & 24,5 \\
Supervisão & Ignorado & 3,0 & 6,4 \\
Normas & Ignorado & 2,3 & 5,3 \\
\hline
\end{tabular}

relação ao CBVE, ainda foi possivel aproveitar, embora parcialmente, as informações coletadas. Para tal, as unidades foram agrupadas segundo o seguinte critério: pelo menos um egresso do curso, nenhum funcionário participante do curso; ausência de qualquer informação a respeito. Esta última categoria incluiu $45 \%$ do total das unidades, refletindo a dificuldade de obtenção da informação.

Considerando que mais de 3.000 profissionais de todos os Estados foram treinados com este material, e que em apenas 228 unidades se encontrou alguns destes, fica a pergunta: onde estariam os demais? Em parte, talvez, distribuídos pelas unidades que não souberam informar, e possivelmente exercendo atividades que pouca ou nenhuma relação têm com o conteúdo dos cursos avaliados. Outra parcela, provavelmente, exerce funções em outros locais não visitados, incluindo o nível central e regional. Além disso, a própria rotatividade dos profissionais, ocasionada pclas precárias condições de trabalho, gera uma situação em que o profissional indicado por detcrminada unidade para o curso muda scu local de urabalho e passa a exercer outras funções, pouco relacionadas ao treinamento reccbido.

Assim, embora a prescnça de egresso do CBVE tenha se correlacionado a um melhor desempenho das unidades, seu papel parece ser muito menor do que o desejado, especialmente quando se considera o volume dos recursos $\mathrm{cm}$ pregados nos treinamentos.
Tabela 4. Percentual de unidades na classe ""2", categorias que as caracterizam e percentual destas categorias no universo total na classe. Brasil, 1985. Classe " 2 " -216 unidades $-22,8 \%$ do total

\begin{tabular}{llrc}
\hline Variável & $\begin{array}{c}\text { Categorias } \\
\text { Caracteristicas }\end{array}$ & $\begin{array}{c}\% \text { no } \\
\text { Universo }\end{array}$ & $\begin{array}{c}\% \text { na } \\
\text { Classe }\end{array}$ \\
\hline Análise de Dados & Ausente & 23,9 & 79,2 \\
Informante & Outras unidades & 19,6 & 53,2 \\
Notificação & Correta & 53,7 & 83,8 \\
Investigação Epid. Presente & 9,8 & 28,2 \\
Nourmas & Presente & 20,5 & 39,3 \\
Boletim Epid. & Presente & 26,7 & 45,4 \\
Instituição & Estado & 34,6 & 53,2 \\
CBVE & Presente & 24,1 & 37,0 \\
PNI & Presente & 69,6 & 81,5 \\
Supervisāo & Presente & 17,2 & 26,4 \\
Unidade Subord. & Presente & 8,9 & 15,7 \\
Dados & & & \\
Demográficos & Presentes & 15,6 & 23,6 \\
Escolarid. Entrev. & Nivel Superior & 71,9 & 79,6 \\
\hline
\end{tabular}

O processo de classificação das unidades apresenta resultados interessantes, resumidos nas Tabelas 3 a 8 .

O grupo "*1", (Tabcla 3) com 188 unidades $(19,8 \%)$, apresenta desempenho precário em relação às atividades básicas da vigilância epidemiológica, não enviando notificaçōes, não registrando os casos de doenças ocorridos e não vacinando. Em relação aos fatores determinantes, a situação é cocrente: a unidade não tem atividade comunitária alguma, desconhece sua área de abrangência ou população adstrita, não recebe boletim epidemiológico e não dispõe das normas de vigilância. É característica do grupo a falta de informação sobre presença de egresso do CBVE.

Os grupos "*2" (Tabela 4) e "*5" (Tabcla 7), somando 280 unidades $(25,6 \%)$ apresentam correto desempenho no conjunto das questōes fundamentais. Ambos os grupos centralizam a notificação de outras unidades de saúde. Em relação aos fatores determinantes, também é semclhante o desempenho. A diferença entre as duas classes é a realização ou não de algum tipo de análise com os dados registrados, observado somente no grupo "*5", com apenas $6,8 \%$ do total de unidades.

O grupo "*6" (Tabcla 8), com 52 unidades $(5,5 \%)$ tem como característica mais marcante negar qualquer conhecimento de casos de diversas doenças de notificação compulsória, o que é surpreendente, considerando a alta prevalência de algumas destas doenças. Este fato, reforçado pela ausência de qualquer outra fonte de informações de casos de doenças, exceto os funcionários da própria unidade sugere que nessas unidades a notificação negativa significa apenas o cumprimento 
Tabela 5. Percentual de unidades na classe "* $3 "$, categorias que as caracterizam e percentual destas categorias no universo total na classe, Brasil, 1985.

Classe * 3 * -107 unidades $-11,3 \%$ do total

\begin{tabular}{llrl}
\hline Variável & $\begin{array}{c}\text { Categorias } \\
\text { Caracteristicas }\end{array}$ & $\begin{array}{c}\% \text { no } \\
\text { Universo }\end{array}$ & $\begin{array}{c}\% \text { na } \\
\text { Classe }\end{array}$ \\
\hline Informante & Variados & 13,9 & 98,1 \\
Investigação Epid. Ausente & 60,4 & 78,5 \\
Instituição & Município & 31,6 & 49,5 \\
PNi & Presente & 69,6 & 85,0 \\
Ativ. Comunitária & Alguma & 37,9 & 54,2 \\
Análise de Dados & Sem registro & 63,5 & 77,6 \\
Ativ. Comunitária & Presente & 11,1 & 20,6 \\
Dados & & & \\
Demográficos & Parciais & 32,7 & 43,0 \\
Unidade Subord. & Ausente & 90,3 & 95,3 \\
Notificaçäo & Correta & 53,7 & 62,6 \\
Boletim Epid. & Presente & 26,7 & 33,6 \\
\hline
\end{tabular}

Tabela 6. Percentual de unidades na classe "4", categorias que as caracterizam e percentual destas categorias no universo total na classe, Brasil, 1985.

Classe " 4 * -321 unidades $-33,9 \%$ do total

\begin{tabular}{llcc}
\hline Variável & $\begin{array}{c}\text { Categorias } \\
\text { Características }\end{array}$ & $\begin{array}{c}\% \text { no } \\
\text { Universo }\end{array}$ & $\begin{array}{c}\% \text { na } \\
\text { Classe }\end{array}$ \\
\hline Informante & $\begin{array}{l}\text { Somente } \\
\text { funcionários }\end{array}$ & 44,3 & 87,5 \\
Análise de Dados & Sem registro & 63,5 & 91,3 \\
Notificação & Irregular & 32,0 & 54,5 \\
Boletim Epid. & Ausente & 72,4 & 82,9 \\
Normas & Ausentes & 77,2 & 86,6 \\
Investigação Epid. Nega existência de & & \\
& casos & 27,2 & 34,9 \\
Instituição & INAMPS & 17,1 & 23,1 \\
Ativ. Comunitária & Nenhuma & 50,8 & 58,3 \\
Unidade Subord. Ausente & 90,3 & 94,1 \\
Dados & Aún & & \\
Demográficos & Nenhum & 51,6 & 57,6 \\
Supervisão & Ausente & 79,9 & 84,4 \\
CBVE & Não dispōe de & 45,3 & 49,2 \\
& informação & & \\
\hline
\end{tabular}

burocrático das normas, não refletindo a inexistência de casos das doenças.

O grupo "*3" (Tabela 5), com 107 unidades $(11,3 \%)$, apresenta um desempenho irregular. Por um lado, tem como fontes de informação entidades e instituições de fora da área da saúde, o que mostra uma prálica de vigilância epidemiológica mais ligada à real ocorrência dos eventos na população. Mas, por outro, não faz investigação cpidemiológica e não apresenta registro de casos.

Por fim, o grupo "*4" (Tabela 6), com 321 uni-
Tabela 7. Percentual de unidades na classe "*5", categorias que as caracterizam e percentual destas categorias no universo total na classe, Brasil, 1985.

Classe ${ }^{*} 5^{*}-64$ unidades $-6,8 \%$ do total

\begin{tabular}{llrc}
\hline Variável & $\begin{array}{c}\text { Categorias } \\
\text { Caracteristicas }\end{array}$ & $\begin{array}{c}\% \text { no } \\
\text { Universo }\end{array}$ & $\begin{array}{c}\% \text { na } \\
\text { Classe }\end{array}$ \\
\hline Análise de Dados & Presente & 6,3 & 93,8 \\
Investigação Epid. Presente & 9,8 & 35,9 \\
Boletim Epid. & Presente & 26,7 & 56,3 \\
Dados & & & \\
Demográficos & Presentes & 15,6 & 40,6 \\
Normas & Presentes & 20,5 & 42,2 \\
Informante & Outras unidades & 19,6 & 37,5 \\
Unidade Subord. & Presente & 8,9 & 21,9 \\
Ativ. Comunitária & Presente & 11,1 & 23,4 \\
Notificação & Correta & 53,7 & 70,3 \\
CBVE & Presente & 24,0 & 39,1 \\
Instituição & Estado & 34,6 & 50,0 \\
Escolarid. Entrev. Nivel Superior & 71,9 & 82,8 \\
\hline
\end{tabular}

Tabela 8. Percentual de unidades na classe " $6 "$, categorias que as caracterizam e percentual destas categorias no universo total na classe, Brasil, 1985. Classe $6^{*}-52$ unidades $-5,5 \%$ do total

\begin{tabular}{|c|c|c|c|}
\hline Variável & $\begin{array}{c}\text { Categorias } \\
\text { Características }\end{array}$ & $\begin{array}{c}\% \text { no } \\
\text { Universo }\end{array}$ & $\begin{array}{l}\% \text { na } \\
\text { Classe }\end{array}$ \\
\hline $\begin{array}{l}\text { Análise de Dados } \\
\text { Investigação Epid. }\end{array}$ & $\begin{array}{l}\text { Nega existência de } \\
\text { casos } \\
\text { Nega existência de }\end{array}$ & 5,6 & 100,0 \\
\hline & casos & 27,2 & 90,4 \\
\hline Notificação & Correta & 53,7 & 78,8 \\
\hline $\begin{array}{l}\text { Instituição } \\
\text { Informante }\end{array}$ & $\begin{array}{l}\text { Estado } \\
\text { Somente }\end{array}$ & 34,6 & 53,8 \\
\hline Dados & funcionários & 44,3 & 59,6 \\
\hline Demográficos & Presentes & 15,6 & 25,0 \\
\hline PNI & Presente & 69,6 & 78,8 \\
\hline Supervisão & lgnorado & 3,0 & 5,8 \\
\hline
\end{tabular}

dades, representa aproximadamente um terço da amostra investigada. O desempenho deste grupo está, no essencial, em desacordo com as normas.

Não hă registro de casos e a notificação é irregular, impontual e sem notificação negativa. São unidades que não têm qualquer atividade comunitäria e desconhecem os dados demográficos de sua área.

Em sintese, frente ao quadro descrito, pode-se afirmar que mais da metade das unidades, constituída pclos grupos "*1" e "*4", têm desempenho precário $\mathrm{cm}$ questões consideradas básicas das normas de vigilância epidemiológica. Menos de um terço, os grupos "*2" e "*5" apresentam desempenho bom, ficando o restante em situação 
intermediária. Além disso, como era esperado, fica evidente a relação dos fatores determinantes selecionados com a prática de vigilância epidemiológica.

\section{Comentários Finais}

Nestes quase 5 anos decorridos desde a visita às unidades, durante o trabalho de campo da pesquisa, diversas mudanças importantes ocorreram na estrutura da prestação de serviços de saúde no país. A partir da $8^{\mathrm{a}}$ Conferência Nacional de Saúde, com a organização dos Sistemas Unificados Descentralizados de Saúde (SUDS), algumas alterações importantes se fizeram sentir. Entre estas, talvez a mais perceptível em nivel dos serviços de vigilância epidemiológica, tenha sido a entrada dos governos municipais de forma mais intensa na área da saúde, com a criação de dezenas de unidades básicas. Este movimento, já então se fazia sentir, através da constatação de que um grande número de unidades, principalmente municipais, tinham sido criadas entre 1983 e 1985, o ano da pesquisa.

Entretanto, as transformações realizadas até aqui ainda não mudaram o funcionamento da alividade de vigilância epidemiológica. A aproximação ocorrida entre os perfis das unidades de diferentes instituições, como as Secretarias Estaduais de Saúde, as Secretarias Municipais de Saúde e o INAMPS, deveu-se principalmente à incorporação da assistência médica, durante o início da década de 80, pelas unidades originalmente voltada para a Saúde Pública. As atividades tradicionalmente exclusivas destas unidades, como a investigação epidemiológica, continuaram especialidades das Secretarias Estaduais de Sáude e de determinadas Secretarias Municipais de Saúde de perfil semelhante, além da Fundação SESP. Assim, é rclevante discutir nas suas linhas básicas como se desenvolvem as atividades de vigilância epidemiológica nas maiores cidades do pais.

A pergunta básica que gostaríamos de responder é: o que realmente influencia o desempenho das unidades na vigilância epidemiológica? Ou seja, se treinamento, supervisāo, e as demais variáveis pesquisadas não são suficientes para responder a esta pergunta, que outros aspectos merecem atenção?

Um aspecto interessante desta avaliação foi observado na análise dos dados relativos ao PNI. Há uma relação entre a capacidade das unidades de calcular os indicadores do PNI e de analisar a distribuição das doenças sob vigilância. Um novo determinante poderia então scr definido: a integração entre as atividades de vigilância epidemio- lógica e de imunizações. Esta, apesar de preconizada nos manuais e treinamentos, dificilmente é implementada.

A importância disto é óbvia: integrar a análise epidemiológica ao desenvolvimento das medidas de controle. A forma como deveria se dar esta integração pode ser sugerida pela análise das situações onde esta integração, ao menos parcialmente, ocorre. Aparentemente o desempenho no programa de imunizaçocs fica fortalecido nos aspectos de programação, avaliação e estratégias, permitindo uma prática mais reflexiva.

Esta constatação, quando extrapolada para o âmbito da vigilância epidemiológica como um todo, lcvou à scguinte pergunta: qual a rclação da vigilância epidemiológica com o restante das atividades dos serviços de saúde, e qual a importância desta relação no desempenho das unidades em vigilância?

A análise histórica da vigilância epidemiológica no Brasil, a partir de sua origem nos programas verticalizados de controle de alguns agravos, aponta os caminhos para esta reflexão ${ }^{11}$. Exceto em documentos de intençōes, as atividades da vigilância epidemiológica no país sempre tiveram carátcr localizado, freqüentcmente reduzindo-se à implantação de medidas de controle definidas extcrnamente ao sistema de saúde do país. Não que tais programas fossem inoportunos. Esta certamente não é a situação da campanha de erradicação da varíola, ou da estruturação do PNI nos moldes definidos pela OPAS. Entretanto, esta atuação desvinculada de uma análise que estivesse sendo feita a partir da realidade das próprias unidades fez com que a vigilância epidemiológica sempre tivesse uma inserção diferenciada, atế nas unidades tradicionalmente responsáveis pela atividade.

Os setores responsáveis pela vigilância epidemiológica nas unidades, e não cstamos nos referindo aos hospitais, onde a vigilância epidemiológica, se presente, limita-se à notificação compulsória, têm, em geral, atribuições que não são discutidas, ou sequer compreendida pelos demais profissionais. Freqüentemente reportam-se somente aos nívcis superiores da estrutura da rede, funcionando de forma inteiramente independente do resto e sem mecanismos de articulação.

O que faltaria, então para que esta integração de fato ocorresse? O processo de municipalização das cstruturas sanitárias, ora em curso, talvez facilite esta transformação, aproximando os responsávcis pela vigilância epidemiológica da realidade municipal. Entretanto, em alguns locais onde se implantam as reformas estruturais no sistema de saúde, repcte-se a dicotomia entre vigilância epidemiológica $\mathrm{e}$ as demais atividades dos serviços, clínicos, laboratoriais e, principalmente, gerenciais. 
As recomendações do Seminário Pcrspectivas da Epidemiologia frente à Reorganização dos Serviços de Saúde ${ }^{14}$ apontam uma questão fundamental situando a vigilância epidemiológica como parte de um sistema de informações $\mathrm{cm}$ saúde. Caberia à vigilância epidemiológica produzir informaçōes que pudessem ser utilizadas pclos responsáveis pela gestão dos sistcmas locais de saúde (SILOS) na programação de suas atividades. Assim, conforme diversas recomendaçöes feitas em seminários e encontros da área, scu campo de atuação naturalmente se ampliaria, abrangendo de imediato as docnças nãotransmissíveis e a monitoração de fatores de risco relacionados às nosologias mais prevalentes ${ }^{14,15}$. Não é concebivel a redução da epidemiologia dos serviços de saúde às doenças transmissíveis, nem o gerenciamento dos serviços e programas scm a informação epidemiológica.

Acreditamos que os resultados da presente pesquisa contribuam para a compreensão do funcionamento das atividades de vigilância epidemiológica e imunizações, tal como ainda são exccutadas nos serviços de saúde, colaborando no desenho de estratégias de superação dos problemas detectados e do próprio modelo de vigilância existente.

CARVALHO, M.S. \& MARZOCCHI, K.B.F. [Assessment of epidemiological surveillance practice in the public health services of Brazil]. Rev. Saúde públ., S. Paulo, 26: 66-74, 1992. The results of a process evaluation of the epidemiological surveillance activities in 948 health units, situated in 98 of the most populated cities of each State in the country are presented. The survey was conducted towards the end of 1985 . The following aspects were analised: information system, data analysis, epidemiological investigation. Institutional insertion, vaccination activities, management aspects and capacitation of the health worker were considered as potential determinants of performance. Data were submitted to correspondence analysis and a process of ascendent hicrarchical classification, using the statistical package "Systeme Portable Pour L' Analise de Données - SPAD". The performance pattern was not found to be homogeneous. Six different classes of epidemiological surveillance practice in the health units were observed. In $53.7 \%$ of the services visited, even the most elementary norms of activity were not complied with. The presence of vaccination activities in the heal th units was associated with beller performance in epidemiological surveillance. The study points to the need to review the epidemiological surveillance model in use in Brazil. It is no longer acceptable to restrict the practice of epidemiology in health services to communicable diseases, now to manage programs and services without epidemiological information.

Keywords: Population surveillance. Program evaluation. Immunization.

\section{Referências Bibliográficas}

1. BORGES, M.V. Enfermagem na vigilância epidemiológica de serviços básicos de saúde: um enfoque de participação. Rio de Janciro, 1980. [Dissertação de Mestrado - Escola Ana Neri de Enfermagem da UFRJ].

2. CLNTRE DE STATISTIQUE ET D'NFORMATIQUE ALIQUEES (CESIA). Systeme portable pour l'analyse des donnćes (SPAD). Paris, 1985. [Mimeografado].

3. ESCOLA NACIONAL DE SAÚDE PÚBLICA. Avaliação do impacto da estratégia de treinamento da SNBS/MS em controle de doenças transmissíveis; relatório final. Rio de Janeiro, 1986. [Mimeografado].

4. FUNDAÇÃO IBGE. Anuório estatístico do Brasil: 1983. Rio de Janciro, 1984.

5. LEBART, L.; MORINEAU, A.; WARWICK, K.M. Multivariate descriptive stalistical analysis: correspondence analysis and related techniques for large matrices. New York, J.Wiley, 1984. (Scries in Probability and Mathematical Statistics).

6. MINISTÉRIO DA SAÚDE. Comitê de Controle de Doenças Transmissívcis. Sistcma Nacional de Controle de Doenças Transmissívcis. Brasilia, 1983. [Mimeografado].

7. MINISTÉRIO DA SAÚDE. Comitê Interorgânico de Controle de Doenças Transmissívcis. Oficina de trabalho para definir o perfil do responsável pela vigilância epidemiológica dos níveis regional, local e periférico do sistema nacional de controle de doenças transmissíveis c ampliação do CBVE; relatório. Brasília, 1984. [Mimeografado].

8. MINISTÉRIO DA SAÚDE. Divisão Nacional de Epidemiologia. Guia de vigilancia epidemiológica. $2^{2}$ ed. Brasília, Centro de Documentação do Ministério da Saúde, 1986. (Série A: Normas e Manuais Técnicos, 21).

9. MINIS'I'ÉRIO DA SAÚDE. Divisão Nacional de Epidemiologia. Manual de procedimentos de vacinaçâo. Brasília, Centro de Documentação do Ministério da Saúde, 1983. (Séric A: Normas e Manuais Técnicos, 15).

10. MINISTLERIO DA SAUDE. Sccretaria Nacional de Açōes Básicas em Saúde. Relatórios das supervisōes às Secretarias de Saúde de Mato Grosso do Sul, Espírito Santo, Maranhão, Amazonas, Paraíba, Roraima, Rio Grande do Norte, Goiás e Alagoas sobre controle de doenças transmissíveis. Brasília, 1984. [Mimeografado].

11. RODRIGUES, B. de A. Fundamentos de administraçấo sanitćria. $2^{\mathrm{a}} \mathrm{ed}$. B rasília, 1979.

12. ROMERO, A. \& VALVERDE, E. Estabelecimento de um sistema integral de vigilância epidemiológica. Bol. Ofic. sanit. panamer., 78: 501-18, 1975.

13. SLCRLETARIA DE SAÚDE DA BAHIA. Serviços de vigilância cpidemiológica nos centros de saúde de Salvador. Salvador, Centro de Desenvolvimento de Recursos Humanos, 1983. [Mimcografado].

14. SEMINÁRIO PERSPLCTIVAS DA EPIDEMIOLOGIA FRENTE À REORGANIZAÇÃO DOS SERVIÇOS DE SAÚDE, Rio de Janciro, 1986. Relatório final. Rio de Janeiro, ABRASCO, 1986. p. 109-27.

15. SEMIN $\Lambda$ RIO SOBRE USOS Y PERSPECTIVAS DE LA EPIDEMIOLOGIA, Buenos Aires, 1983; informe final. Washington, DC, Organizacion Panamericana de la Salud, 1984. (OPAS - Publicación IPNSP 84-47).

Recebido para publicação em 23/5/1991 Reapresentado em 7/1/1992 Aprovado para publicaçäo em 10/2/1992 
ANEXO

Variáveis, símbolos utilizados e descrição das categorias

\begin{tabular}{|c|c|c|}
\hline Variáveis & Simbolos & Explicação de cada categoria \\
\hline $\begin{array}{l}\text { Informaçőes } \\
\text { de doenças } \\
\text { transmissiveis }\end{array}$ & $\begin{array}{l}\text { INFM } \\
\text { INFO } \\
\text { INFS } \\
\text { INFI }\end{array}$ & $\begin{array}{l}\text { nenhum profissional da unidade informa casos de doenças } \\
\text { transmissiveis } \\
\text { apenas profissionais da unidade informam casos de doenças } \\
\text { transmissíveis } \\
\text { informaçōes de doenças transmissiveis colhidas em instituiçōes } \\
\text { comunitárias } \\
\text { informaçōes recolhidas em outras unidades de saúde } \\
\text { lgnorado }\end{array}$ \\
\hline $\begin{array}{l}\text { Envio } \\
\text { de Notificação }\end{array}$ & $\begin{array}{l}\text { NOTS } \\
\text { NOTM } \\
\text { NOTN } \\
\text { NOTI }\end{array}$ & $\begin{array}{l}\text { notifica de acordo com as normas } \\
\text { notifica irregularmente } \\
\text { não envia notificaçāo } \\
\text { ignorado }\end{array}$ \\
\hline $\begin{array}{l}\text { Análise } \\
\text { de dados }\end{array}$ & $\begin{array}{l}\text { SANA } \\
\text { NANA } \\
\text { RANA } \\
\text { FANA } \\
\text { IANA }\end{array}$ & $\begin{array}{l}\text { análise mínima de dados realizada (pessoa, tempo, lugar) } \\
\text { nenhuma análise de dados } \\
\text { não há registro na unidade } \\
\text { não houveram casos de doenças transmissiveis no periodo pes- } \\
\text { quisado } \\
\text { Ignorado }\end{array}$ \\
\hline $\begin{array}{l}\text { Ficha } \\
\text { de investigação } \\
\text { epidemiológica }\end{array}$ & $\begin{array}{l}\text { SFIC } \\
\text { NFIC } \\
\text { FFIC } \\
\text { IFIC }\end{array}$ & $\begin{array}{l}\text { na ficha de investigação epidemiológica } \\
\text { não há ficha de investigação epidemiológica } \\
\text { nāo houveram casos de doenças transmissiveis no periodo pes- } \\
\text { quisado } \\
\text { ignorado }\end{array}$ \\
\hline $\begin{array}{l}\text { Instituição } \\
\text { mantenedora }\end{array}$ & $\begin{array}{l}\text { SES } \\
\text { SMS } \\
\text { INPS } \\
\text { HOSP }\end{array}$ & $\begin{array}{l}\text { Secretaria Estadual de Saúde } \\
\text { Secretaria Municipal de Saúde } \\
\text { INAMPS } \\
\text { unidade hospitalar, independente da instituição mantenedora }\end{array}$ \\
\hline $\begin{array}{l}\text { Existência } \\
\text { de dados } \\
\text { demográficos }\end{array}$ & $\begin{array}{l}\text { DEMO } \\
\text { DEMM } \\
\text { DEMN } \\
\text { DEMI }\end{array}$ & $\begin{array}{l}\text { dados demográficos com distribuição por sexo e faixas etárias } \\
\text { dados demográficos incompletos } \\
\text { ausência de dados demográficos } \\
\text { ignorado }\end{array}$ \\
\hline $\begin{array}{l}\text { Existência } \\
\text { de relação } \\
\text { com a comunidade }\end{array}$ & $\begin{array}{l}\text { COMO } \\
\text { COMM } \\
\text { COMN } \\
\text { COMI }\end{array}$ & $\begin{array}{l}\text { diversas formas de relaçăo comunitária (reuniōes, jornais, pales- } \\
\text { tras) } \\
\text { pelo menos um tipo de trabalho comunitário } \\
\text { nenhum trabalho comunitário } \\
\text { ignorado }\end{array}$ \\
\hline Vacinaçăo & $\begin{array}{l}\text { SPNI } \\
\text { NPNI }\end{array}$ & $\begin{array}{l}\text { aplica as vacinas do PNI } \\
\text { não aplica as vacinas do PNI }\end{array}$ \\
\hline $\begin{array}{l}\text { Presença } \\
\text { de egresso } \\
\text { do CBVE }\end{array}$ & $\begin{array}{l}\text { SCBV } \\
\text { NCBV } \\
\text { ICBV }\end{array}$ & $\begin{array}{l}\text { pelo menos um egresso de Curso Básico de Vigilância Epide- } \\
\text { miológica/MS } \\
\text { nenhum egresso do CBVE na unidade } \\
\text { ignorado }\end{array}$ \\
\hline $\begin{array}{l}\text { Supervisão } \\
\text { em vigilância } \\
\text { epidemiológica }\end{array}$ & $\begin{array}{l}\text { SSVE } \\
\text { NSVE } \\
\text { ISVE }\end{array}$ & $\begin{array}{l}\text { recebeu visita de supervisão abrangendo vigilância epidemio- } \\
\text { lógica } \\
\text { não recebeu supervisão abrangendo vigilância epidemiológica } \\
\text { ignorado }\end{array}$ \\
\hline $\begin{array}{l}\text { Dispōe } \\
\text { de normas } \\
\text { escritas }\end{array}$ & $\begin{array}{l}\text { SNOR } \\
\text { NNOR } \\
\text { INOR }\end{array}$ & $\begin{array}{l}\text { dispōe de normas escritas de vigilância epidemiológica } \\
\text { não dispōe de normas escritas de vigilância epidemiológica } \\
\text { ignorado }\end{array}$ \\
\hline $\begin{array}{l}\text { Recebe } \\
\text { boletim } \\
\text { epidemiológico }\end{array}$ & $\begin{array}{l}\text { SBOL } \\
\text { NBOL } \\
\text { IBOL }\end{array}$ & $\begin{array}{l}\text { recebeu boletim epidemiológico } \\
\text { nāo recebeu boletim epidemiológico } \\
\text { ignorado }\end{array}$ \\
\hline
\end{tabular}

